\title{
Due ritratti della moglie di Giobbe (Gb 2,9-10)
}

Dos Retratos de La MUJeR DE Job (Job 2,9-10).- Tampoco la mujer de Job (Job 2,910), como sucede con otros personajes menores de las historias de la Biblia hebrea, juega un papel insignificante en la historia de su marido, sino que es relevante para entender mejor la intención del autor. En la interpretación tradicional, principalmente basada en el Texto Masorético (TM), ella estaba encuadrada dentro de un esquema que le atraía el juicio negativo de la crítica, fruto tal vez de una visión misógina de matriz judeo-helenística, y aparecía como hostil a Job, el justo sufridor, por la aversión a la fe incondicional de este. Ahora queremos revisar aquí esta controvertida figura, primero de todo a la luz de los testimonios de las dos ramas de la tradición textual hebrea (TM) y griega (Old Greek $=\mathrm{OG}$ ). En el primer caso, las palabras de la mujer de Job suenan como una invitación a su marido a maldecir a Dios, como responsable de sus injustos sufrimientos; en el segundo, sin embargo, se muestran como una exhortación a proclamar ante Dios su dolor inocente, aun a costa de ser castigado con la muerte. Encontramos así dos retratos diferentes, si no opuestos, de la mujer de Job, cuyo papel en la historia de Job se define negativa o positivamente según el arquetipo presupuesto.

Palabras Clave: Job; la mujer de Job; retratos; Texto Masorético; Old Greek; misoginia.

Two Portraits of Job's Wife (Job 2:9-10).- Even Job's wife (Job 2:9-10), like the minor characters of the Hebrew Bible stories, does not play at all an insignificant role in the story of her husband, but she is however functional to better understand the author's design. In the traditional interpretation, based mostly on the Masoretic Text (MT), she was caught in a pattern that drew the negative opinion of the critics, perhaps the result of a misogynistic view, of Judeo-Hellenistic matrix, and then she appeared as hostile to Job, the righteous sufferer, for the aversion to the unconditional faithfulness of this man. Now, here we wanted to re-read this controversial figure, first of all starting from the received witnesses by both streams of the textual tradition, Jewish (MT) and Greek (Old Greek $=\mathrm{OG}$ ). In the first case, the words of his wife sound like an invitation to Job to curse Yahweh, responsible for his unjust suffering; in the second, however, they urge him to cry out to God his innocent suffering, even if he should be at the cost of being punished

\footnotetext{
giancarlo.toloni@unicatt.it
} 
by death. So, two different portraits of woman - if not opposites - are derived from that, and her role in Job's story is defined negatively or positively, according to the archetype that is supposed.

Keywords: Job, the Righteous Sufferer; Job's Wife; Portraits; Masoretic Text; Old Greek; Misogyny.

\section{IL PROBLEMA CRITICO}

Non deve sorprendere, all'interno dell'attuale dibattito scientifico, la presenza di un interesse crescente per i personaggi secondari delle narrazioni bibliche ${ }^{1}$. Essi, infatti, pur non svolgendo un ruolo di primo piano rispetto ai protagonisti, rivestono un'importanza non certo marginale nell'intreccio abbozzato dall'autore, dato che spesso integrano l'azione dei personaggi primari e ne scandiscono i passaggi nodali. L'attenzione che in sede critica si sta via via riservando ai personaggi minori -specialmente femminili- ${ }^{2}$, secondo le suggestioni della moderna narratologia, è quindi orientata a sottolineare che la loro funzione va colta nella complementarietà -talvolta pur in formale contrapposizione- tra ruoli secondari e principali. In tale prospettiva, perciò, lo studio delle presenze minori nei racconti diventa significativo in vista della corretta valutazione della psicologia dei protagonisti, oltre che ai fini della comprensione dello sviluppo della narrazione.

In particolare, nella vicenda di Giobbe - presentata nel prologo e nell'epilogo del libro omonimo con numerose affinità con quella di Tobi- ${ }^{3}$ la funzione svolta dalla moglie è di fatto antagonistica rispetto a lui. È assodato il senso del comportamento di Giobbe, volto a ribadire l'etica tradizionale: il successo, con il premio della virtù sottoposta alla

\footnotetext{
Cf. per es. il recente contributo di A. NEPI, Dal fondale alla ribalta. I personaggi secondari nella Bibbia ebraica. Presentazione di J. L. SKA (Bologna 2015). In assenza di altre indicazioni, le traduzioni dei passi che verranno esaminati in questo studio sono da attribuirsi a me.

${ }^{2}$ Cf. per es. P. P. A. FunARI, «Mulheres na Bíblia Hebraica», in Santas e Sedutoras, as heroinas na Bíblia Hebraica, ed. E. SERra Braga, vol. I (São Paulo 2010) pp. 11-13.

${ }^{3}$ Per un esame delle analogie tematiche e strutturali dei due racconti, grazie alle quali è possibile risalire alla leggenda antica che li anima, si veda G. Toloni, La sofferenza del giusto. Giobbe e Tobia a confronto (Brescia 2009) pp. 38-58. Cf. anche J. VermeYLEn, Job, ses amis et son Dieu. La légende de Job et ses relectures postexiliques (Leiden 1986) pp. 29-33.
} 
tentazione, alla fine arride a chi si mantiene fedele, quale conferma della prospettiva della retribuzione del bene e del male; meno chiaro è invece il significato dell'atteggiamento della moglie. Varie ipotesi sono state proposte circa l'origine e il valore della sua menzione in Gb 2,9-10 a partire dalla spiegazione più nota, che stigmatizza tale raffigurazione come improntata a misoginia in quanto proveniente dalla tradizione sapienziale giudeo-ellenistica ( $c f$. Pr 5,7), di cui rifletterebbe la prospettiva religiosa ${ }^{5}$, fino alle interpretazioni moderne, perlopiù in chiave femminista, che individuano nel passo suddetto tratti ed accenti propri dell'ambito socio-politico ${ }^{6}$.

Si profilano quindi due opposte linee di ricerca, che lasciano intravedere due immagini divergenti della moglie di Giobbe: l'una, di stampo tradizionale, è connotata negativamente; l'altra, di recente formulazione, più positivamente. Entrambe, tuttavia, sono tratteggiate sulla base del Testo Masoretico (TM). In questo studio, perciò, prima di entrare nel vivo del dibattito critico e proporre un'interpretazione personale, sembra opportuno studiare attentamente ambedue i rami della tradizione di $\mathrm{Gb}$ 2,910, ebraico e greco, rappresentati, nell'ordine, dal TM e dalla LXX antica (Old Greek, d'ora in poi OG). In effetti lo studio preliminare di questi due testimonia permetterà di cogliere la rispettiva presentazione che vi si dà del ruolo della donna nell'ora in cui ella si misura, sia pur di riflesso, con la sfida rivolta all'integrità del marito. Quindi, in un lavoro successivo, si valuteranno in sintesi i dati raccolti con l'analisi del testo, e si procederà a un confronto delle due immagini così delineate, sulla cui base si potrà proporre più opportunamente qualche considerazione di carattere esegetico circa Gb 2,9-10.

\footnotetext{
${ }^{4}$ Una rassegna puntualissima è offerta da E. O. Gravett, «Biblical Response: Past and Present Retellings of the Enigmatic Mrs. Job», Biblical Interpretation 20 (2012) pp. 97-125.

${ }^{5}$ Cf. per es. F. Vattioni, Per il testo di Giobbe (Napoli 1996) p. 2, n. 15. Secondo G. Ravasi, Giobbe. Traduzione e commento (2a ed., Roma 1984) p. 313, la moglie di Giobbe rappresenterebbe «l'incarnazione della religione ipocrita ed interessata».

${ }^{6}$ Su questa prospettiva si veda per es. N. DAS Neves e L. A. S. Rossi, «A mulher de Jó, um grito de protesto: uma releitura do livro de Jó sob a perspectiva da teologia feminista», Estudos Teológicos 51 (2011) pp. 116-126; Ch. MAIER e S. ScHrOER, «Job: Questioning the Book of the Righteous Sufferer», in Feminist Biblical Interpretation: A Compendium of Critical Commentary on the Books of the Bible and Related Literature, eds. L. SchotTroff, M.-T. WACKER e M. RuMSCHEIDT (Grand Rapids, MI, 2012) pp. 221-239.
} 
2. IL TESTO EBRAICO

Nel TM si legge ${ }^{7}$ :

9

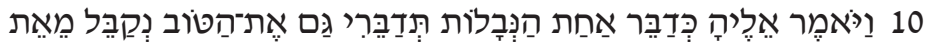

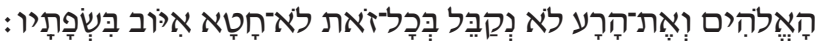

9 «Allora sua moglie gli disse: "Resti saldo ancora nella tua integrità? (a) / Maledici Dio e muori!" (b).

10 Ma egli le rispose: "Hai parlato come parlerebbe una stolta! Se da Dio accettiamo il bene, perché non dovremo accettare il male?" (a) / In tutto ciò Giobbe non peccò con le sue labbra (b)».

Il passo non presenta problemi testuali, come conferma anche l'apparato critico $^{8}$. Vi si riporta l'invettiva della moglie di Giobbe (v. 9), intenta a scuotere il marito perché si ricreda di fronte alle ingiuste sofferenze che hanno messo a dura prova la sua fedeltà. Giobbe tuttavia rimane fedele a Yhwh: le parole con cui apostrofa la moglie (v. 10) suonano come un rimprovero per lei, ma nel contempo mirano a educarla a comprendere l'etica tradizionale animata dalla fede.

Studieremo criticamente questo brano anzitutto mediante l'analisi filologico-linguistica, senza peraltro tralasciare l'esame dei contributi più significativi della storia dell'interpretazione, che si profila ricca e articolata'.

\subsection{Analisi filologica}

Già a una prima lettura del testo si presenta un problema esegetico interessante. L'esortazione che la moglie rivolge a Giobbe (v. 9b) è co-

${ }^{7}$ G. Gerlemann (ed.), «Iob», in Biblia Hebraica Stuttgartensia ... (BHS), eds. K. Elliger e W. Rudolph (5a ed., Stuttgart 1997) pp. 1227-1274: 1229.

${ }^{8}$ BHS, apparato critico, p. 1229.

${ }^{9}$ C. A. Newsom, The Book of Job: A Contest of Moral Imaginations (New YorkOxford 2003) p. 3 definisce Giobbe come «Polyphonic Text» per la molteplicità dei suoi generi letterari (pp. 11-15) e delle metodologie utilizzabili per lo studio critico (pp. 6-11). 
stituita da due imperativi decisi e drastici nella loro portata. Tuttavia, se il senso del secondo («muori!») è fuori discussione ${ }^{10}$, dato che potrebbe essere inteso, se non come una vera e propria istigazione al suicidio ${ }^{11}$, certamente come un invito a prepararsi alla morte quale punizione per la sua blasfemia, invece quello del primo suscita qualche dubbio che nasce dalla difficoltà di comprendere con chiarezza se la moglie abbia ingiunto al marito: «benedici» o «maledici» Dio! In effetti nel testo figura il ver-

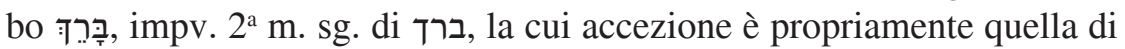
«benedire». Tuttavia nel libro di Giobbe (in particolare in 1,5.11; 2,5.9) il verbo ברך assume talora il senso di «maledire», divenendo un sinonimo di קלל, che al qittel significa appunto «ingiuriare o screditare con un linguaggio diffamatorio» ${ }^{12}$; nel nostro passo, avendo per oggetto Yhwh, poté sembrare scandaloso agli scribi, che quindi lo corressero con un eufemismo per «maledire» ${ }^{13}$. C'è anche però chi ritiene che, più che di un tiqqûn sôf rîm ${ }^{14}$, o di una correzione successiva di un copista, debba trattarsi di una scelta lessicale imputabile all'autore stesso del libro ${ }^{15}$.

${ }^{10}$ Con D. J. A. CuInes, Job 1-20, vol. I (Dallas, TX, 1989) p. 5, esso indica le dirette conseguenze del primo imperativo, quindi non può ridursi a una mera annotazione temporale col senso di «prima di morire».

${ }^{11}$ Secondo A. Weiser, Giobbe. Traduzione e commento (2a ed., Brescia 2003) p. 55, le parole della donna, se non costituiscono propriamente un'esortazione al suicidio, sono pur sempre ispirate dai sentimenti contrapposti di compassione e disperazione. Sulla possibile «compassione» concorda anche H. Gross, Giobbe (Brescia 2002) p. 25.

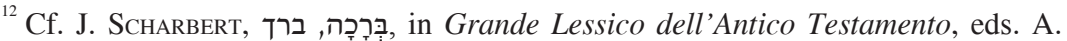
Catastini e R. Contini vol. I (Brescia 1988; ed. orig. G.J. Botterweck e H. RingGren [eds.], Theologisches Wörterbuch zum Alten Testament [Stuttgart 1973]), pp. 1645-1712: 1682.

${ }^{13}$ L. Alonso Schökel, Dizionario di ebraico biblico (Cinisello Balsamo 2013) pp.

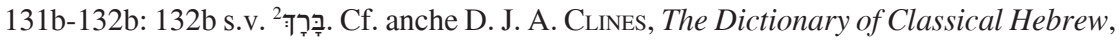
vol. II (Sheffield 1995) pp. 267a-271b: 270a, s.v. ברך I; L. Koehler e W. Baumgartner, Hebräisches und aramäisches Lexikon zum Alten Testament vol. I ( $3^{\text {a }}$ ed., Leiden 1967), pp. 152b-153b: 153b, s.v. ברך II. J. P. FoKKeLman, The Book of Job in Form: A Literary Translation with Commentary (Leiden 2012) p. 200 ritiene che la carica fortemente emotiva delle parole della moglie abbia suggerito all'autore di sostituire il verbo «maledire» con «benedire» anche in altri tre passi del prologo di Giobbe, cioè in 1,5; 2,5; 3,1. Sull'eufemismo cf. anche M. H. PoPE, Job: Introduction, Translation, and Notes $\left(3^{\mathrm{a}}\right.$ ed., $2^{a}$ rist., Garden City, NY, 1974) p. 22.

${ }^{14}$ Cf. E. Dhorme, A Commentary on the Book of Job (Nashville, TN, 1984) pp. 4-5.

${ }^{15}$ S. H. Blank, «The Curse, the Blasphemy, the Spell, the Oath», Hebrew Union College Annual 23 (1950-51) pp. 73-95: 83-85. Così anche N. C. HABEL, The Book of Job: 
Del resto molti commentatori -soprattutto moderni-confermano che, alla luce del contesto, il senso dell'espressione è quello di un incitamento alla ribellione, e che in tal modo la moglie mirerebbe a strappare a Giobbe una dura parola contro Dio, quasi una maledizione, prima che egli perisca. Forse, proprio per questa istigazione alla bestemmia, ci si sarebbe potuto aspettare che la moglie, che esce di scena a questo punto, vi rientrasse nell'epilogo -e con lei anche il $\operatorname{satan}^{16}$, che ora sparisce a sua volta- per subire da parte di Yhwh la giusta condanna di tale ardire. Ma l'autore, come rileva Vermeylen $^{17}$, non dice più nulla della sofferenza della donna, colpita, come Giobbe, dalla sventura.

Si deve notare, tuttavia, che, anche intesa alla lettera nel significato di «benedire», l'esortazione della moglie non muterebbe sostanzialmente di senso, assumendo semmai una sfumatura sarcastica ${ }^{18}$, volta a far rilevare la noncuranza di Yhwh nei confronti della pietas dei giusti, che continuano a benedirlo pur subendo l'ennesima pena a vaglio della loro fedeltà. Del resto, come suggerisce Magdalene ${ }^{19}$, è forse anche possibile che all'interno dell'area semantica di ברך non esista una netta distinzione tra le due opposte accezioni di «maledire» e «benedire».

In definitiva è evidente che il testo lascia indeterminato ${ }^{20}$ il significato specifico che ha qui l'imperativo di ברך, che può suonare come una maledizione, nel senso di «difendersi da una sventura», oppure come una benedizione, nel senso di «lodare ciò che è lodevole», forse anche con

A Commentary (Philadelphia, PA, 1985) p. 88; M. WeIss, The Story of Job's Beginning: Job 1-2, A Literary Analysis (Jerusalem 1983) p. 30.

${ }^{16}$ Per ragioni pratiche, il nome del tentatore sarà qui riportato con la grafia di uso corrente (satan), anziché con la corretta traslitterazione (śățān).

${ }^{17}$ Vermeylen, Job, ses amis et son Dieu, p. 8.

${ }^{18}$ Sul senso dell'esclamazione cf. anche J. Trebolle [Barrera] e S. Pottecher, Job (Madrid 2011) p. 118.

${ }^{19}$ F. R. Magdalene, «Job's Wife as Hero: A Feminist-Forensic Reading of the Book of Job», Biblical Interpretation 14 (2006) pp. 209-258: 209, n. 2, sulla scorta di M. Cheney, Dust, Wind, and Agony: Character, Speech, and Genre in Job (Stockholm 1994) pp. 62-67. Così anche E. van WoLDE, «The Development of Job: Mrs. Job as Catalyst», in Feminist Companion to Wisdom Literature, ed. A. BrenneR (Sheffield 1995) pp. 201-221: 203-204.

\footnotetext{
${ }^{20}$ Cf. Maier e Schroer, «Job», p. 233.
} 
sfumatura ironica ${ }^{21}$. Pertanto, con Seow ${ }^{22}$, si potrebbe pensare che il narratore, intuita l'ambiguità dell' invettiva della donna, abbia accennato così anche alla possibilità di Giobbe di rimproverarla ingiustamente, lasciando intravedere la sua intima inclinazione a maledire Yhwh.

Qual è allora il vero senso delle parole che la moglie rivolge a Giobbe (v. 9b)? Alonso Schökel $^{23}$ ha sottolineato con pertinenza che, proprio nel suo incitare il marito a maledire Dio, ella raccoglie l'istigazione del $s a$ tan, agendo quasi da sua «complice inconsapevole» ${ }^{24}$. Nelle parole della donna affiora quindi la prospettiva di una religiosità interessata, «condizionata al comportamento di Dio», da benedirsi da parte dell'uomo se questi ne riceve $\mathrm{i}$ benefici, e da maledirsi se Yhwh gli appare invece malefico nei suoi confronti. Quindi -ella sembra concludere-, se Giobbe, il giusto, ora deve morire, colpito da una prova terribile e immeritata, tanto vale che possa gustare almeno la consolazione ultima della vendetta impotente, maledicendo il suo carnefice ${ }^{25}$. Pertanto la moglie è ispirata da due sentimenti: anzitutto affetto profondo e sincero per il marito, in virtù del quale si immedesima quasi nella sua sorte, poi ribellione a un Dio che appare crudele ai suoi occhi. È quindi evidente, come fa ben notare Alonso Schökel, che ella non è solo istigatrice alla maledizione, perché il suo obbiettivo è anche e anzitutto quello di proclamare l'innocenza di

\footnotetext{
${ }^{21}$ Per questo E. M. Good, In Turns of Tempest: A Reading of Job (Stanford, CA, 1990) p. 200 suggerisce che l'esortazione della moglie in $\mathrm{Gb}$ 2,9b potrebbe egualmente essere intesa come un congedo ( «Di' addio a Dio e muori!»); una ribellione («Rinfaccia ogni cosa a Dio e accettane le conseguenze di morte»); un incoraggiamento («Tieniti saldo nella tua integrità, resisti nella tua devozione sino alla morte»); o un peccato («Maledici Dio e sii liberato dalla tua sofferenza»).

${ }^{22}$ Ch. L. SEow, Job 1-21: Interpretation and Commentary (Grand Rapids, MI, 2013) p. 305.

${ }^{23}$ Alonso SchöKel e Sicre Díaz, Giobbe, pp. 109-110.

${ }^{24}$ CuInes, Job, pp. 50-51 sottolinea l'ambiguità del ruolo della moglie, tanto che il suo intervento potrebbe apparire in prima istanza come un'ulteriore tentazione per il marito. Ella non accetta che possa esistere una pietà religiosa gratuita e fondata puramente sul timore di Yhwh. D'altra parte la donna non ha dubbi sull'integrità di Giobbe, quindi anche l'invito a disporsi ad accettare la morte, che rimane in sé una suggestione empia, non la rende empia a sua volta perché ispirato da parole umane e a tutto vantaggio per lui.

${ }^{25}$ Anche T. Longman III, Job (Grand Rapids, MI, 2012) p. 90 è convinto che Giobbe appaia innocente agli occhi della moglie, che perciò, a differenza dei tre amici, non gli consiglia affatto di pentirsi, bensì lo invita a maledire Dio che presumibilmente lo punirà, perché considera la morte migliore delle sue attuali sofferenze.
} 
Giobbe, difendendola dalla «ingiustizia» di Yhwh: «si Dios es injusto, no tiene derecho a la bendición del hombre» ${ }^{26}$.

D'altra parte, le poche parole che la donna pronuncia $(2,9 \mathrm{~b})$ sono le stesse già del satan $(2,5)$; ma, paradossalmente, nel contempo esse riprendono (2,9a) anche quelle di Yhwh $(2,3 b)^{27}$. Questo complica l'analogia, e ad un tempo conferma la fisionomia della moglie come personaggio intermedio tra Giobbe e i suoi veri tentatori, di cui ella è solo un'eco nell'intreccio del racconto.

Nella prima parte della sua risposta (v. 10a) il marito la paragona a una «stolta» (lett.: «una delle donne stolte»). In che senso? Due sono le accezioni fondamentali di נָָָ :

1. «Abietto, vile, villano». Si tratta dell'epiteto tradizionale della letteratura sapienziale (in Pr 17,7 è l'opposto di נָדָ', «nobile»; in 30,22 equivale ad עֶֶֶ, «servo») per indicare l'antitesi dell'uomo sapiente e religioso. Il נָָָל è quindi l' «abietto» che «indirizza contro Yhwh propositi aberranti» (Is 32,6). Il fedele, invece, sa che il dolore non è necessariamente una prova dell'ostilità di Dio, ma il segno di un suo progetto libero e misterioso che l'uomo deve accogliere. Infatti nella prospettiva del prologo Yhwh non vuole la sofferenza fine a se stessa, ma come prova della fedeltà.

2. «Stolto, insensato, dissennato», o anche «mentecatto, stupido, idiota». In Dt 32,6 נָָָ è l'opposto di חדָָ, «assennato», e designa il popolo «non intelligente», che non comprende la storia; nel Sal 14,1 lo è chi «dice invece nel suo cuore: Dio non c'è», negando la sua azione nel mondo.

In $\mathrm{Gb}$ 2,10 perciò figura la seconda accezione ${ }^{28}$, come conferma il contesto; in questo modo l'autore intende sottolineare che la moglie di

\footnotetext{
${ }^{26}$ Alonso SchöKel e Sicre Díaz, Giobbe, p. 110.

${ }^{27}$ Sulle parole della moglie si veda anche W. Vogels, «Job’s Empty Pious Slogans (Job 1,20-22; 2,8-10)», in The Book of Job, ed. W. A. M. BEUKEN (Leuven 1994) pp. 369-376: 373.

${ }^{28}$ Con Alonso Schökel, Dizionario, pp. 522-523, s.v. נָָָ Cf. anche Alonso SchÖKEl e Sicre Díaz, Giobbe, p. 110, dove si esclude per il passo in esame la precedente accezione. Clines, Dictionary, vol. V (Sheffield 2001) assegna al vocabolo tre sfumature di significato sostanzialmente affini, rendendo l'espressione di Gb 2,10 (אַחת הַ:ְּבָלוֹת) con «one of foolish women (una donna stolta)» (p. 593b, s.v. נָבד I), «one of the outcast women (una donna abietta)» (p. 594a, s.v. נָָָ II), e «one of the low-born women (una donna di umili origini)» (p. 594a-b, s.v. נָּב IV). In Koehler, BAumgartner e StAmm, Lexikon, vol. III ( $3^{\text {a }}$ ed., Leiden 1983) pp. 626b-627a, s.v. נבל I, si annota un'accezione che esprime un
} 
Giobbe, nella sua compassione e tenerezza per il marito così duramente provato, non riesce a intuire il senso di quel che sta succedendo. Tale è la sua stoltezza o dissennatezza, in linea con la tradizione sapienziale dove questo comportamento è l'antitesi della Sapienza. La donna stolta è perciò quella che «distrugge la sua casa con le sue mani» $(\operatorname{Pr} 9,13)$. Rinnegare Dio e il suo progetto, a volte difficile da capire, è la stoltezza per eccellenza (Pr 9,1-6.13-18; Sal 14,1)

Radermakers ${ }^{30}$ osserva giustamente che per spiegare queste parole ardite della moglie, che pare incitare Giobbe alla blasfemia, non c'è bisogno di accusare l'autore di misoginia: «questa povera donna parla come noi quando siamo collocati davanti all'eccesso del male». Ai suoi occhi -e a quelli di chi non crede-, rinnegare Yhwh è l'unico modo per porre fine a sofferenze tanto inaudite e ingiuste. Giobbe però non acconsente all'esortazione e non pecca con le parole, cioè «con le sue labbra» non pronuncia la «bestemmia»: resiste alla tentazione del suicidio, che -secondo la prospettiva sapienziale suddetta- sarebbe stato autentica «stoltezza». Così rimane solo nella sua scelta; la moglie esce di scena, e il piano del satan registra un altro insuccesso.

\subsection{Alcune interpretazioni tradizionali}

Il brano ha largamente ispirato l'esegesi cristiana, giudaica e musulmana, con varie riletture della figura femminile qui rappresentata e della sua reazione alle sollecitazioni del $\operatorname{satan}^{31}$.

Nella tradizione cristiana primitiva ${ }^{32}$, in particolare, spicca la designazione della moglie di Giobbe come diaboli adiutrix, «collaboratrice del

atteggiamento negativo verso Yhwh, cioè «Tor, Gottesleugner (stolto, ateo)», spiegando che potrebbe figurare in $\mathrm{Gb} 2,10$.

${ }^{29}$ Anche SEOW, Job 1-21, p. 305 intende נָבָ in questo senso, spiegando che esso non designa l'assenza di facoltà intellettuali («foolish»), bensì una carenza di carattere teologico o etico («impious»). $40-41$

${ }^{30}$ J. RAdermakers, Il libro di Giobbe. Dio, l'uomo e la sapienza (Bologna 1999) pp.

${ }^{31}$ Una presentazione dettagliata della storia dell'interpretazione della vicenda di Giobbe e dei suoi antagonisti si trova in Longman, Job, pp. 39-44.

${ }^{32}$ Sull'esegesi cristiana relativa alla figura della moglie di Giobbe rimando a K. Low, 
diavolo», proposta da Agostino ${ }^{33}$ che vi coglie un richiamo a Eva, la prima tentatrice. Mediante una lettura allegorica, con qualche prossimità alla considerazione tipologica, egli fa notare l'esistenza di alcune interessanti analogie tra $i$ due racconti biblici in cui esse figurano. In effetti, come l'invito alla trasgressione è rivolto da Eva ad Adam, dopo essere stata vittima delle lusinghe del serpente, così le parole della moglie di Giobbe rivelano che anch'ella di fatto aveva subito l'adulazione del satan. Tuttavia, a differenza di Adam, Giobbe non cede e si mantiene fedele. Secondo Agostino, quindi, la moglie di quest'ultimo è anzitutto «dedita al servizio del diavolo, non alla consolazione del marito (seruata ad ministerium diaboli, non ad solatium mariti)» ${ }^{34}$.

Gregorio Magno $^{35}$ sviluppa la lettura allegorica paragonando la fedeltà di Giobbe a una fortezza di cui il satan tenta l'assalto servendosi della moglie come di una scala. Avendo già sperimentato la via attraverso la quale Adam si lascia ingannare, cioè Eva, il tentatore ora ricorre similmente alla moglie di Giobbe; così egli «conquistò l'animo della sposa, scala del marito (Occupavit animum conjugis, scalam mariti)». Gregorio sottolinea quindi che con la rettitudine delle sue parole Giobbe diede una lezione alla moglie, che il serpente aveva istigato a parlare perversamente.

Già Giovanni Crisostomo ${ }^{36}$-che fa di Giobbe il typus christiani o anche la figura Christi-aveva confrontato Adam con Giobbe circa la sedu-

The Bible, Gender and Reception History: The Case of Job's Wife (London-New York 2013) pp. 31-47; cf. anche Ch. L. SEow, «Job's Wife, with Due Respect», in Das Buch Hiob und seine Interpretationen. Beiträge zum Hiob-Symposium auf dem Monte Verità, eds. Th. KrÜger, M. Oeming, K. Schmid e Ch. Uehlinger (Zürich 2007) pp. 351-373: 351-354.

${ }^{33}$ Augustinus, Sermo De Symbolo ad Catechumenos III 10 (256): «Voluit mulier illa, diaboli adiutrix, non mariti consolatrix, persuadere blasphemiam». Cf. J. B. BAUER (ed.), Sermo ad catechumenos de symbolo, in De fide rerum invisibilium; Enchiridion ad Laurentium de fide et spe et caritate; De catechizandis rudibus... eds. M. P. J. vAN DEN Hout et al. (Corpus Christianorum Series Latina [CCSL] 46; Turnholti 1969) p. 193. Sul valore di questo richiamo letterario cf. anche G. KaISER e H.-P. MAthys, Il libro di Giobbe (Brescia 2015) p. 27.

${ }^{34}$ Augustinus, Enarrationes in Psalmos XCVII 6. Cf. E. Dekkers e J. Fraipont (eds.), Enarrationes in Psalmos LI-C (CCSL 39; 2a ed., Turnholti 1990) p. 1374.

${ }^{35}$ Gregorius Magnus, Moralia in Iob VIII 12. Cf. M. Adriaen (ed.), Moralia in Iob Libri I-X (CCSL 143; Turnholti 1979) p. 122.

${ }^{36}$ Ionnnes Chrysostomus, Homiliae III de Imbecillitate Diaboli: De diabolo tentatore, Homilia III 3. Cf. PG 49, pp. 268-270: 269 = II 107-108.114 secondo l'edizione critica 
zione a cui entrambi furono soggetti, cogliendovi varie differenze. Nel primo caso l'insidia fu ordita con le sole parole, nel secondo con i fatti; inoltre il serpente era un servo, un suddito, la moglie invece una convivente, una collaboratrice. Tuttavia, mentre Eva fu sconfitta dal servo e suddito, Giobbe «né la consorte, né la collaboratrice lo poté vincere (oủ $\delta \dot{\varepsilon}$

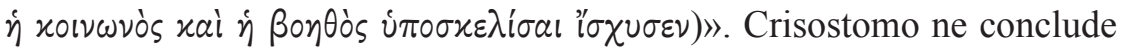
che in tal modo la moglie, invece di chiudere la bocca al demonio o comunque di tacere, nella sua stoltezza «fornì a costui una grande occasione

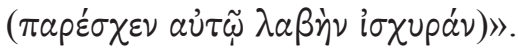

Calvino, infine, designa la donna come organum Satanae ${ }^{37}$, «strumento di Satana»-immagine già di Tommaso d'Aquino ${ }^{38}$-, precisando che il demonio le aveva risparmiato le sventure e le prove arrecate al marito per potere continuare, tramite lei, la sua sfida nei confronti di quest'ultimo.

Negli scritti della tradizione giudaica la moglie di Giobbe non è più anonima $^{39}$. Nel Targum di Giobbe, in particolare, le si assegna un nome, cioè Dina, che la farà assimilare alla figlia di Giacobbe, così chiamata ${ }^{40}$.

di A. Peleanu (ed.), Jean Chrysostome, Homélies sur l'impuissance du diable (Sources Chrétiennens 560; Paris 2013) p. 180. Sul confronto della moglie di Giobbe con Eva in Crisostomo si veda C. BRoc, «La femme de Job dans la prédication de Jean Chrysostome», Studia Patristica 37 (2001) pp. 396-403.

${ }^{37}$ Ioannes Calvinus, In Librum Jobi Conciones IX. Cf. Johannis Calvini in Librum Jobi Conciones. Cum Praefatione Theodori Bezae (Genevae 1593) p. 36a.

${ }^{38}$ Thomas de Aquino, Expositio super Iob ad litteram II 2: «dixit autem illi uxor sua, quam solam Diabolus reliquerat ut per eam viri iusti mentem pulsaret qui per feminam primum hominem deiecerat». Cf. Sancti Thomae de Aquino Opera omnia iussu impensaque Leonis XIII P.M. edita, Tomus XXVI: Expositio super Iob ad litteram, ed. A. DondaINE (Romae 1965) s. pp. (in formato elettronico). Cf. anche Thomas Aquinas, The Literal Exposition on Job: A Scriptural Commentary Concerning Providence. Translated by Anthony Damico and Interpretive Essay and Notes by Martin D. Yaffe (Atlanta 1989) p. 94.

${ }^{39}$ Testam. Job 1,6; Ps.-Phil. 8,7-8; BT Baba Bathrā 15a. Cf. Gen. Rab. 57,4; 76,9. Cf. anche SEOw, Job 1-21, p. 304 e Pope, Job, p. 23, dove si aggiunge che in BT Baba Bathrā $15 \mathrm{~b}$ e nel Targum di Giobbe Dina figura come seconda moglie di Giobbe. Sulle varie ipotesi cf. LONGMAN, Job, p. 89, n. 41.

${ }^{40}$ Poiché il Targum antico di Giobbe è perduto e quello pervenuto è tardo, qui ci si avvarrà dell'edizione di B. Walton (ed.), Biblia Sacra Polyglotta vol. III (Londini 1657; rist. Graz 1964), sez. $1^{\mathrm{a}}$, p. 6, $2^{\mathrm{a}}$ parte, confrontandola con quella di L. Díez Merino (ed.), Targum de Job. Edición Principe del Ms. Villa-Amil n. 5 de Alfonso de Zamora (Madrid 1984) p. 187. Sulla figura di Giobbe e dei suoi antagonisti nei Targumim rimando allo 


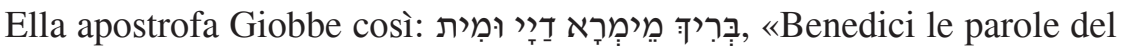
Signore, e muori». La maggior parte degli studiosi sembra incline a considerare la sollecitazione all'esordio («Benedici») come un eufemismo («Maledici»), in conformità con quanto s’è visto per il TM corrispettivo. Tuttavia va anche ricordato che, in un interessante studio, O'Connor ${ }^{41}$ propone una valutazione sostanzialmente benevola dell'esortazione di Dina a Giobbe. Egli suggerisce di intenderla in senso letterale, senza più bisogno di ricorrere a una lettura eufemistica. Su questa interpretazione si è espressa Mangan con due interventi. Anzitutto ${ }^{42}$ ella presenta la tesi di O’Connor, verso la quale mostra attenzione, ma sottolinea lo sforzo esegetico che essa comporta, cosa che rischia forse di sembrare un punto debole dell'argomentazione addotta. In un contributo successivo ${ }^{43}$ Mangan riprende l'analisi testuale, suggerendo però una diversa interpretazione. Sulla base di alcuni precisi riscontri linguistici, legati alla disposizione sintattica degli elementi dei costrutti del passo in esame, rispetto agli altri tre del Targum in cui ricorre בִּרִ (Gb 1,5; 1,11;2,5), ella preferisce pensare che anche in 2,9 tale forma verbale costituisca un eufemismo per «maledici», e che quindi non possa essere resa alla lettera, avendo una chiara sfumatura di disprezzo. Del resto nei tre passi suddetti il Targum traduce l'eufemismo del TM con parole e formule sprezzanti, e anche quando il verbo conserva un simile valore traslato in 2,9 lascia intuire dal contesto che deve essere egualmente considerato in senso dispregiativo. Pertanto Mangan ritiene che non si può intendere benevolmente l'esortazione di Dina nel Targum.

Nel resto del passo continua l'accordo della versione aramaica con il TM.

L'identificazione della moglie di Giobbe con Dina, figlia di Giacobbe, pare risalire a Rabbi ben Kahana (Midr. Gen. Rab. 19,12). Tale interpre-

studio rigoroso di C. MANGAN, «The Interpretation of Job in the Targums», in The Book of Job, ed. W. A. M. BEUKEN (Leuven 1994) pp. 267-279.

${ }^{41}$ D. J. O'Connor, Job, His Wife, His Friends and His God (Dublin 1995) pp. 25-27.

${ }^{42}$ C. Mangan, «The Attitude to Women in the Prologue of Targum Job», in Targumic and Cognate Studies: Essays in Honour of Martin McNamara, eds. K. J. CATHCART e M. MAHER (Sheffield 1996) pp. 1-110: 108.

${ }^{43}$ C. Mangan, «Blessing and Cursing in the Prologue of Targum Job», in P. V. M. Flesher (ed.), Targum and Scripture: Studies in Aramaic Translation and Interpretation in Memory of Ernest G. Clarke (Leiden 2002) pp. 225-229: 229. 
tazione fu forse suggerita dal midrash costituito dal richiamo del termine נְבָלוֹת (Gb 2,10), «stolte»-detto, come s'è visto, delle donne cui viene paragonata la moglie di Giobbe per le sue parole- al corradicale נְבָּלָה, «oltraggio», con cui in Gen 34,7 si designa l'atto scellerato del rapimento e della violenza subiti da Dina da parte di Sichem, figlio di Camor l'Ivveo (cf. Gen 34,2) ${ }^{44}$. Quindi l'esegesi rabbinica -come Agostino- vede nella moglie di Giobbe una ripresa della prima tentatrice (Eva), e sottolinea anche la fedeltà di Giobbe che, a differenza di Adam, non accondiscese alla provocazione della moglie ${ }^{45}$.

Anche nella tradizione musulmana ${ }^{46}$ viene assegnato alla moglie di Giobbe un ruolo significativo all'interno della vicenda di quest'ultimo: la sua funzione traspare già dal nome che qui viene attribuito alla donna, Raḥma, cioè «misericordia». Ella infatti è descritta come premurosa e totalmente dedita al marito che, da musulmana osservante, soccorre prontamente, portandogli dell'acqua perché possa resistere nella prova, stremato e provato com'è anche nel fisico ${ }^{47}$. Pope ${ }^{48}$ menziona inoltre un passo del Corano (XXXVIII 43) in cui si potrebbe cogliere un rimprovero di Giobbe alla moglie, in particolare nelle parole con cui gli amici lo inviterebbero a fare un fascio (probabilmente di giunchi) e a percuoter(la?) così da non infrangere il suo giuramento; egli spiega questo passaggio -in verità poco chiaro- supponendo che in un momento di rabbia Giobbe avesse giurato di dare alla moglie un centinaio di sferzate se fosse guarito. Ma i commentatori musulmani sono vaghi su quale fosse effettivamente la colpa della donna, fino a ipotizzare genericamente che ella potrebbe forse essere stata per troppo tempo fuori casa per una commissione.

${ }^{44}$ Così anche Clines, Job 1-20, p. 53.

${ }^{45}$ Cf. J. Theodor e Ch. Albeck (eds.), Midrash Bereshit Rabba: Critical Edition with Notes and Commentary (Jerusalem 1965) ad loc. Il commento alla Genesi noto come Genesi Rabbah (o anche Bereshit Rabbah) è uno dei midrashim esegetici più antichi e importanti, databile al V sec. d.C. (G. STEMBERGER, Introduzione al Talmud e al Midrash [Roma 1995] pp. 384-385).

${ }^{46}$ Una presentazione del dialogo di Giobbe con la moglie secondo la tradizione musulmana si trova nel commento alla Sura XXI 83 di G. SALE, The Koran (London 1889) pp. 247 ss., riportato da POPE, Job, pp. 22-23.

${ }^{47}$ Cf. anche Radermakers, Il libro di Giobbe, p. 40.

${ }^{48}$ Pope, Job, p. 22. 


\section{LA TRADIZIONE TESTUALE GRECA}

\subsection{La versione dei $L X X$}

Nell'edizione critica curata da Ziegler ${ }^{49}$ il passo è riportato come segue:

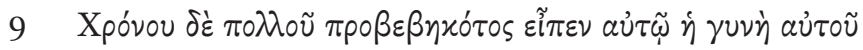

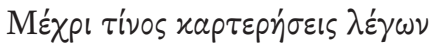

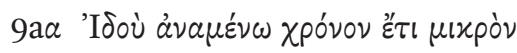

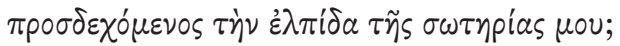

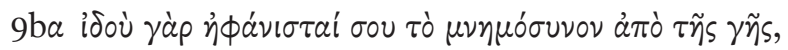

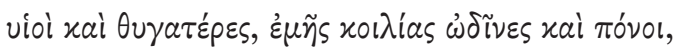

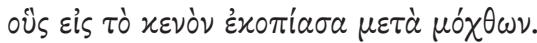

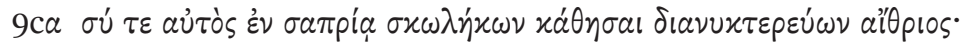

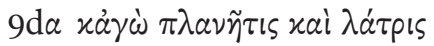

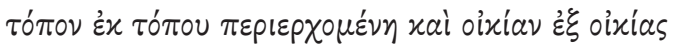

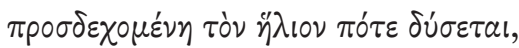

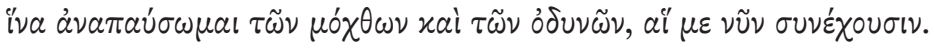

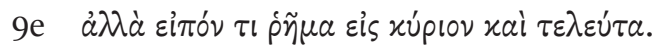

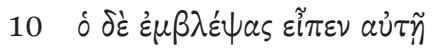

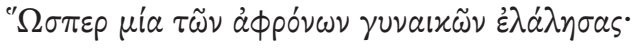

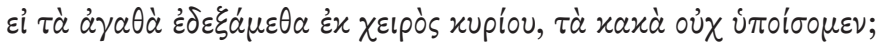

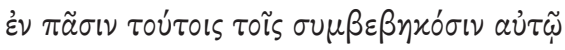

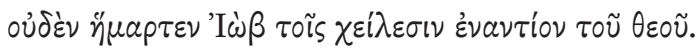

${ }^{49}$ J. ZIEGLER (ed.), Septuaginta. Vetus Testamentum Graecum Auctoritate Academiae Scientiarum Gottingensis editum vol. XI/4: Iob, (Göttingen 1982) pp. 218-220. 
9 «Molto tempo dopo, gli disse sua moglie:

"Fino a quando persevererai ${ }^{50}$, dicendo:

$9 \mathrm{a} \alpha$ 'Ecco, sto in attesa ancora per un po'

affidandomi alla speranza ${ }^{51}$ della mia salvezza?'.

$9 \mathrm{~b} \alpha$ Vedi come è svanito dalla terra il tuo ricordo,

i figli e le figlie, le doglie del mio ventre e gli affanni,

con cui ho penato invano tra gli sforzi.

$9 \mathrm{c} \alpha$ E tu te ne stai seduto tra la putredine di vermi, e passi la notte all' aria aperta,

$9 \mathrm{~d} \alpha$ mentre io, raminga e serva,

me ne vado di luogo in luogo e di casa in casa,

aspettando che il sole finalmente tramonti

per potere avere tregua dagli affanni e dalle pene che ora mi opprimono.

9e Ma di' una parola al Signore ${ }^{52}$, e muori!".

10 Ma egli, volgendole lo sguardo, replicò:

"Hai parlato come una donna stolta:

se dalla mano del Signore abbiamo accettato il bene, non dovremo

sopportare il male?".

In tutto ciò che gli era accaduto,

Giobbe non commise [alcun] peccato con le [sue] labbra dinanzi al Signore».

\footnotetext{
${ }^{50}$ In questo contesto il verbo potrebbe essere reso liberamente con «sopporterai con rassegnazione / con pazienza»; tuttavia si è preferita qui una traduzione più letterale. Così anche J. M. Cañas Reíllo, «Libro de Job», in La Biblia Griega. Septuaginta, III: Libros poéticos y sapienciales, eds. N. Fernández Marcos e M. ${ }^{a}$ V. Spottorno Díaz-Caro (Salamanca 2013) p. 428, che rende con «vas a estar», e C. E. Cox, Iob, in A New English Translation of the Septuagint and the Other Greek Translations Traditionally Included Under That Title (NETS), eds. A. Pietersma e B. G. Wright (New York-Oxford 2007) pp. 667-696: 671b, che traduce «How long will you persist».

${ }^{51}$ CAÑas Ré́llo, «Libro de Job», p. 428 rende liberamente con «mientras conservo la esperanza».

${ }^{52}$ Cf. Cox, Iob (versione NETS), p. 671b: «Now say some word to the Lord». Invece CaÑas Reíllo, «Libro de Job», p. 429 traduce «Pero, di alguna palabra contra el Señor».
} 
Come si vede nei LXX, il testo dei vv. 9-10 è ben più lungo del corrispettivo nel TM, infatti presenta un ampliamento considerevole a causa dell'inserzione di alcuni stichi (v. 9a-e), della cui natura ci si occuperà in seguito.

Anzitutto si nota che il brano pone vari problemi testuali, come confermano le numerose varianti annotate in apparato critico $^{53}$. Tuttavia si tratta perlopiù di divergenze lessicali ${ }^{54} \mathrm{o}$ grafiche ${ }^{55}$, che non infirmano quindi la qualità del testo edito da Ziegler. Seguono sinonimi della lezione testuale ${ }^{56}$, oppure sostituzioni di tempi ${ }^{57}, \operatorname{modi}^{58}$ e diatesi verbali ${ }^{59}$, mutamenti dei preverbi ${ }^{60}$ e delle preposizioni ${ }^{61}$ che reggono i sostantivi, o anche scambi del plurale col singolare e/o viceversa ${ }^{62}$. Talora la variazione è di natura sintattica e riguarda la funzione logica di un vocabolo delle proposizioni ${ }^{63}$, oppure la trasformazione di una frase in una forma verbale equivalente ${ }^{64}$, o la ripetizione del verbo di un emistichio anche nella seconda parte di questo ${ }^{65}$, quasi in una sorta di parallelismo

${ }^{53}$ ZiEGLER, Septuaginta, pp. 218-220, apparato critico I, nn. ai vv. 9-10.

${ }^{54}$ Per es.: $\varepsilon \omega \varsigma \tau$. anziché Mé $\chi \rho ı \tau$. (v. 9b).

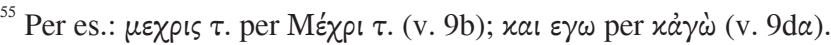

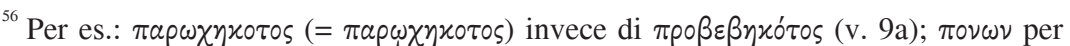

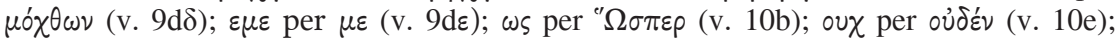

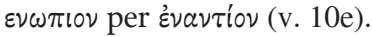

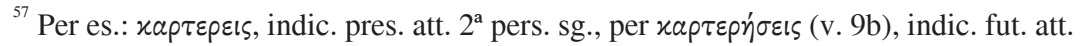
$2^{\mathrm{a}}$ pers. sg.

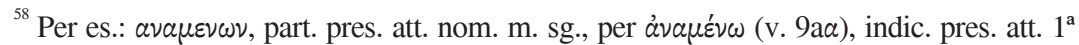

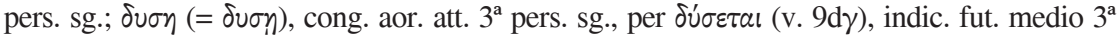

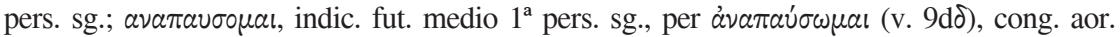
medio $1^{\text {a }}$ pers. sg.; $\varepsilon ı \pi \omega \nu$, part. pres. att. nom. m. sg., per ciiróv (v. 9e), impv. aor. att. $2^{\text {a }}$ pers. sg.

${ }^{59}$ Per es.: $\delta \cup \sigma \varepsilon l$, indic. fut. att. $3^{\text {a }}$ pers. sg., per $\delta \dot{\sigma \varepsilon \varepsilon \tau \alpha l ~(v . ~} 9 \mathrm{~d} \gamma$ ), indic. fut. medio $3^{\mathrm{a}}$ pers. sg.

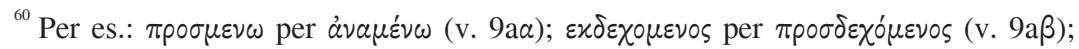
$\pi \varepsilon \rho\llcorner\varepsilon \chi 0 v \sigma เ \nu$ per $\sigma u \nu \varepsilon ́ \chi 0 u \sigma เ \nu$ (v. $9 \mathrm{~d} \varepsilon$ ).

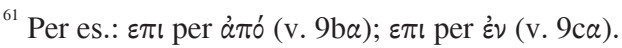

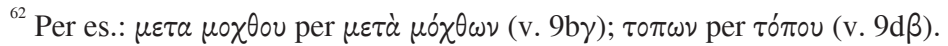

${ }^{63}$ Per es.: $\pi \lambda \alpha \nu \omega \mu \varepsilon \nu \eta$ per $\pi \lambda \alpha \nu \eta \tilde{\tau} \tau \varsigma$ (v. $\left.9 \mathrm{~d} \alpha\right)$; integrazione di $\tau \cup \gamma \chi \alpha \nu \circ v \sigma \alpha$ dopo $\lambda \alpha \dot{\tau} \tau$ เ (v. $9 \mathrm{~d} \alpha)$.

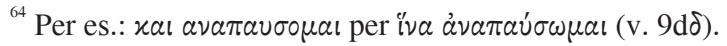

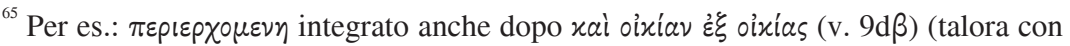
variatio $\mu \varepsilon \tau \alpha \beta a เ v 0 v \sigma \alpha$ o $\delta เ \varepsilon p \chi о \mu \varepsilon \nu \eta)$. 
suggerito forse dallo stile poetico del brano, talvolta anche con l'integrazione di qualche parola per armonizzare lo stico col precedente o col successivo $^{66}$.

A parte, invece, stanno alcune lezioni alternative apprezzabili in sé, infatti le novità che esse propongono potrebbero avere qualche ripercussione sul senso dello stico in cui ricorrono; esse meritano quindi la nostra

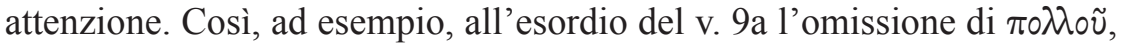
«di molto», che dà all'intera proposizione a cui il termine appartiene

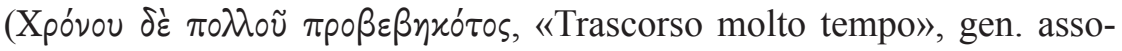
luto di valore temporale) una determinazione più generica, venendo cioè a significare «trascorso del tempo», o anche «dopo qualche tempo». Di

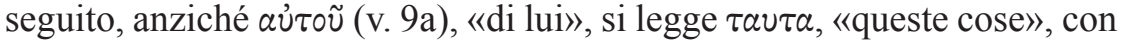
la differenza che il pronome determinativo non è più gen. di specifica-

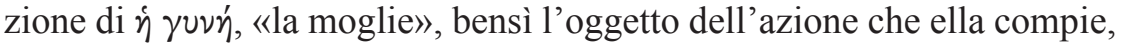

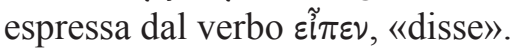

Nel v. 9ba, alla lezione del testo $\dot{\phi} a ́ v ı \tau \tau$ l, «è sparito», indic. perf.

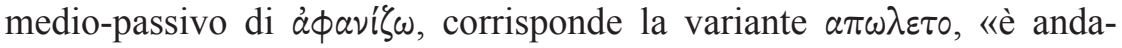
to perduto, si è dileguato, è svanito», indic. aor. medio intransitivo di à $\hat{o}^{\lambda} \lambda u \mu \iota$ (migliore certamente della lezione con la forma attiva e transitiva $\alpha \pi 0 \lambda \omega \lambda \varepsilon \nu$, «ha rovinato»), con cui si esprime forse con maggior efficacia il venir meno del ricordo della vita di Giobbe per la perdita dei figli e delle figlie.

Variazione lessicale più sostanziale è forse oঠuves, «dolori, strazi, pene», al posto di $\omega \delta \tilde{\iota \nu \varepsilon \varsigma, ~ « d o g l i e » ~(v . ~ 9 b \beta), ~ c h e ~ t u t t a v i a ~ s e m b r a ~ p r e f e r i-~}$ bile in quanto più pertinente al contesto. Altrettanto meno propria appare

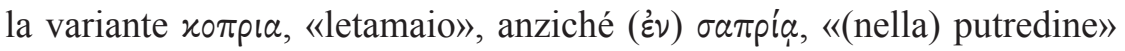
(v. 9c $\alpha$ ), come precisa il gen. di specificazione successivo $\sigma \varkappa \omega \lambda \eta \dot{\eta} \kappa \omega \nu, ~ « \mathrm{di}$ vermi» (v. 9c $\alpha)$.

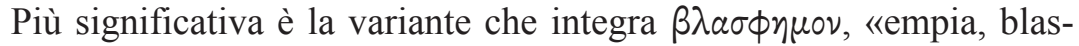
fema», dopo $\hat{\eta} \tilde{\mu} \alpha$, «parola» (v. 9e), e così pure quella che legge $\pi \rho \circ$, «contro», invece di cis, «verso» (v. 9e), con ogni evidenza volte entrambe a specificare che l'esortazione che la moglie rivolge a Giobbe è finalizzata a fargli pronunciare una maledizione esplicita contro Dio.

\footnotetext{
${ }^{66}$ Per es.: $\tau \omega \nu \pi \varepsilon \rho \iota \varepsilon \chi 0 \nu \sigma \omega \nu \mu \varepsilon$ per $\tau \tilde{\omega} \nu$ ỏ $\delta \nu \nu \tilde{\omega} \nu$ (v. $\left.9 \mathrm{~d} \delta\right)$.
} 
Una minor precisione lessicale rivela invece la variante $\cup \pi о \mu \varepsilon \nu о \mu \varepsilon \nu$,

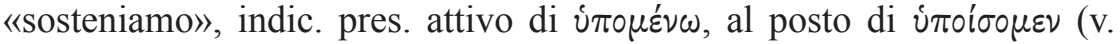

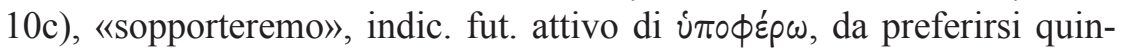
di perché sottolinea meglio la perseveranza e la capacità di tollerare il male. Più appropriata parrebbe forse la variante tou xupiov, «del Signo-

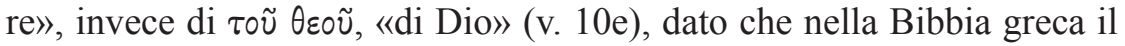
termine xúplos è usato solitamente per rendere il tetragramma sacro del nome divino (Yhwh), mentre $\theta$ cós è solo un epiteto funzionale che indica la natura divina, infatti traduce אֵל / אֶלהִים; ma è pur vero che i due vocaboli talora sono intercambiabili. E del resto, come precisa Fernández $\operatorname{Marcos}^{67}$, è assodato che il traduttore originario di Giobbe, rendendo dall' ebraico in greco con finalità letterarie più che letterali, ellenizzò e unificò i vari nomi di Dio ricorrenti nel libro riducendoli a uno solo, variabile tra xúplos e $\theta$ cós.

In definitiva anche le varianti in apparenza più significative in realtà non sono preferibili perlopiù alla lezione del testo edito da Ziegler.

\subsection{Da OG al testo greco ecclesiastico}

Si tratta ora di appurare che valore possiede la versione greca di Giobbe del passo in esame ai fini della restituzione dell'originale ${ }^{68}$.

Gli orientamenti critici principali si possono ricondurre sostanzialmente a due: quello di Siegert ${ }^{69}$-in linea con gli studi di Orlinski ${ }^{70}$,

${ }^{67}$ N. Fernández Marcos, «The Septuagint Reading of the Book of Job», in The Book of Job, ed. W. A. M. Beuken (Leuven 1994) pp. 251-266: 257.

${ }^{68}$ Sul dibattito critico odierno cf. M. WITTE, «The Greek Book of Job», in Das Buch Hiob und seine Interpretationen. Beiträge zum Hiob-Symposium auf dem Monte Verità, eds. Th. Krüger, M. Oeming, K. Schmid e Ch. Uehlinger (Zürich 2007) pp. 36-41. Sulla trasmissione del greco e le varie edizioni attestate cf. pp. 33-35.

${ }^{69}$ F. SIEGERT, Zwischen Hebräischer Bibel und Altem Testament. Eine Einführung in die Septuaginta (Münster 2001) p. 97.

${ }^{70}$ H. M. OrLINSKY, «Studies in the LXX of the Books of Job, I: An Analytical Survey of Previous Studies», Hebrew Union College Annual [HUCA] 28 (1957) pp. 53-74; «Studies in the LXX of the Books of Job, II: The Character of the LXX Translation», HUCA 29 (1958) pp. 229-271; «Studies in the LXX of the Books of Job, III: On the Matter of Anthropomorphism», HUCA 30 (1959) pp. 153-167; 32 (1961) pp. 239-268; «Studies in the LXX of the Books of Job, IV: The Present State of the Greek Text of Job», HUCA 33 (1962) 
per il quale il greco di Giobbe rappresenterebbe una forma testuale ebraica più antica, tale da anticipare influssi masoretici; quindi quello di $\operatorname{Cox}^{71}$ e Fernández Marcos ${ }^{72}$, propensi a credere invece che la Vorlage del greco di Giobbe potrebbe essere molto simile al $\mathrm{TM}^{73}$. In particolare, circa il passo che qui interessa, Cook $^{74}$ suggerisce un'interpretazione intermedia tra $\mathrm{i}$ due indirizzi generali suddetti, sulla quale però si debbono avanzare alcune riserve. Egli infatti non considera gli stichi supplementari del v. 9a-e come delle aggiunte, dovute a un intervento di poco seriore, né come attestazioni di una Vorlage ebraica diversa dal TM, ma come frutto del lavoro del traduttore originario del greco antico.

Per comprendere meglio i limiti di tale ipotesi è opportuna qualche precisazione circa lo stato degli studi sul greco di Giobbe $e^{75}$. Per la critica è assodato che il testo di OG era di un sesto più breve dell'ebraico trasmesso nel receptus (cioè che aveva circa 390 righe in meno del TM); inoltre, si trattava della versione della Bibbia ebraica meno letterale tra quelle pervenute nel testo ecclesiastico greco (la cosiddetta LXX). Oggi non è più sostenibile la tesi dell'esistenza di un originale ebraico breve di Giobbe, reso da OG, di cui il TM sarebbe un ampliamento successivo. Del resto nei manoscritti qumranici non si trova alcuna documentazione di questa ipotesi; anzi, Qumran preserva parti di testo ebraico assente da OG. Così anche la maggior parte delle versioni fornisce una testimonianza al testo lungo di Giobbe. C'è ragione di credere perciò che non siamo

pp. 119-151; «Studies in the LXX of the Books of Job, V: The Hebrew Vorlage of the LXX of Job: The Text and the Script», HUCA 35 (1964) pp. 57-78, e 36 (1965) pp. 37-47.

${ }^{71}$ C. E. Cox, «Methodological Issues in the Exegesis of LXX Job», in VI Congress of the International Organization for Septuagint and Cognate Studies, ed. C. E. Cox (Jerusalem 1986) pp. 78-89: 80.

${ }^{72}$ Fernández Marcos, «The Septuagint Reading», p. 254; e Introduction to the Greek Versions of the Bible (Boston-Leiden 2000; rist. 2001) p. 80.

${ }^{73}$ Cf. anche WitTe, «The Greek Book», pp. 36-37.

${ }^{74} \mathrm{~J}$. Cook, «Are the Additions in LXX Job 2,9a-e to be deemed as the Old Greek text?», Biblica 91 (2010) pp. 275-284, e, più in generale, «Aspects of Wisdom in the Texts of Job (Chapter 28) - Vorlage(n) and/or Translator(s)», Old Testament Essays, N.S. 5 (1992) pp. 26-45.

${ }^{75}$ Seguo la presentazione di SEOw, Job 1-2, pp. 6-8 e Cox, Iob (versione NETS), p. $671 b$. 
in presenza di due diverse forme testuali, una rappresentata dall'ebraico reso da OG e l'altra da quello della tradizione masoretica, ma che la Vorlage ebraica di OG sia sostanzialmente simile al TM. Due sono quindi i problemi conseguenti.

1) Le omissioni. Cosa manca in OG rispetto al TM? Perlopiù la seconda parte di un distico, la cui assenza rischia di pregiudicare la comprensione del testo; oppure un passo difficile da rendere o teologicamente problematico, e in tal caso OG parrebbe una semplificazione di qualche ridondanza dell'ebraico, pervenuta invece nel TM. Ciò conferma quindi che la Vorlage ebraica di OG non doveva essere più breve del TM, perché non si capirebbe il motivo per cui qualcuno potrebbe averla ampliata, rendendola così più difficile. Ciò soprattutto laddove le omissioni in OG hanno reso il testo molto più fluido e chiaro. Ecco perché sembra preferibile pensare che la Vorlage di OG fosse sostanzialmente simile al TM, sebbene vi siano anche varianti originali in OG, e alcune di esse si dimostrino superiori al TM. Ciò porta a concludere, con Fernández Marcos ${ }^{76}$, che le divergenze e le abbreviazioni nel testo pervenuto dei LXX rispetto al TM sono dovute perlopiù alle tecniche del traduttore.

2) Le integrazioni. Le righe assenti da OG erano invece presenti nella altre traduzioni greche, a partire da Teodozione (II sec. d.C.). Quando Origene (III sec. d.C.) volle tentare di colmare queste lacune, fece riferimento al testo greco predisposto da quest'ultimo ${ }^{77}$; così contrassegnò con un asterisco l'inizio di ogni integrazione, rispetto a OG, e con un metobelo la sua conclusione. Ora, a differenza di OG, il testo di queste integrazioni era reso in un greco molto letterale. Origene poi indicò con un obelo l'inizio delle espressioni assenti dall'ebraico ma presenti in OG, e la loro fine con un metobelo. Il risultato è il cosiddetto «testo ecclesiastico greco» di Giobbe, detto anche LXX, ad uso della Chiesa per la liturgia.

\footnotetext{
${ }^{76}$ Fernández Marcos, «The Septuagint Reading», p. 254.

${ }^{77}$ Sul lavoro critico di Origene relativo al greco di Giobbe rimando all'importante monografia di P. J. Gentry, The Asterisked Materials in the Greek Job (Atlanta, GA, 1995) pp. 2-10.
} 


\subsection{L'aggiunta del v. 9a-e}

Per quanto attiene all' aggiunta di Gb 2,9a-e ${ }^{78}$, è evidente che non può trattarsi -come vorrebbe Cook- del frutto del lavoro del traduttore originario di $\mathrm{OG}$, che è naturalmente di difficile individuazione e complessa valutazione. In effetti la prima traduzione in greco $(\mathrm{OG})$ è un'opera letteraria con una propria validità, non solo in rapporto all'ebraico che essa rende. Del resto l'attenzione dei critici qui non deve mirare a risalire all'ebraico originale o alle intenzioni dei traduttori, bensì a studiare il significato di OG e le modalità della sua ricezione tra i giudei grecofoni, ai quali fu trasmesso un testo greco del tutto comprensibile senza dovere ricorrere all'ebraico corrispettivo ${ }^{79}$.

Neppure si può dire che il v. 9a-e costituisca un'integrazione vera e propria, avvenuta cioè molto più tardi della traduzione di $\mathrm{OG}$, dato che esso non figura tra i materiali contrassegnati con asterischi da Origene ${ }^{80}$. È più logico pensare quindi che si tratti di un'aggiunta incorporata poco dopo in $\mathrm{OG}$, e che perciò sia frutto di un intervento midrashico con finalità esplicative, visto che la sua inserzione non apporta al testo novità sostanziali. Il v. 9a-e è perciò parte integrante di OG, di cui costituisce un'espansione appena successiva alla sua traduzione, e non rappresenta un'altra Vorlage ebraica. Fernández $\operatorname{Marcos}^{81}$, concordando sulla natura midrashica di tale aggiunta, fornisce un'importante indicazione sul suo lessico. Egli sottolinea infatti che, mentre nel TM la risposta drastica della moglie di Giobbe è contenuta nel solo v. 9, in OG essa ha la forma di un lungo lamento elaborato nel v. 9a-e mediante un vocabolario diverso rispetto al resto del libro biblico, indizio almeno di un'incorporazione successiva di questi versetti supplementari. Quindi, come le altre aggiunte a OG (19,4b-c; 36,28c-f; 40,4a-c), anche questa non assume un significato sostanziale, ma indubbiamente costituisce un'autorevole attestazione indiretta che il traduttore seguì una Vorlage non troppo dissimile dal TM.

\footnotetext{
${ }^{78} \mathrm{Di}$ fondamentale importanza per lo studio del problema che qui interessa è certamente il contributo puntualissimo e ben documentato di FERnÁNDEZ MARCOS, «The Septuagint Reading», pp. 261-266.

${ }^{79}$ Fernández Marcos, «The Septuagint Reading», p. 252.

${ }^{80}$ Gentry, The Asterisked Materials, pp. 15-31.

${ }^{81}$ FernándeZ MARCOS, «The Septuagint Reading», p. 261.
} 
Di conseguenza le divergenze di OG da quest'ultimo sono frutto degli interventi letterari e lessicali del traduttore, che agì spesso da interprete dell'ebraico, anziché limitarsi a renderlo nella sua letteralità.

Ziegler ha pubblicato il testo di OG nella sua edizione critica conservando gli asterischi origeniani per indicare le integrazioni successive (suscitando così la reazione di A. Pietersma) $^{82}$; di questi segni diacritici, tuttavia, non c'è traccia nelle versioni moderne (per esempio la NETS, la traduzione spagnola e quella tedesca) ${ }^{83}$, dove non si utilizza nemmeno il corsivo per indicare tali aggiunte bensì le si trascrive col carattere tondo. In effetti nello studio critico si deve prendere in esame il solo testo breve del greco di Giobbe, non le integrazioni tarde di Origene, perché tali espansioni sono state adattate al TM e riflettono il testo ecclesiastico, non OG. Tuttavia nell'edizione di Ziegler gli stichi del v. 9a-e non sono contrassegnati con gli asterischi degli Hexapla origeniani, quindi non possono essere considerati integrazioni successive ma espressioni originali di $\mathrm{OG}$, anche se dovute a una mano secondaria.

Allo stato attuale degli studi non è ancora ben chiaro il senso dell'aggiunta del v. 9a-e. È fuori discussione però che essa è frutto di un' attività esegetica, dato che il versetto viene ampliato e rielaborato sulla base di dettagli impliciti in esso, quindi gli stichi supplementari ne costituiscono un articolato doppione esplicativo ${ }^{84}$. Si tratterà semmai di verificare attentamente quante lezioni di Ziegler sono attribuite a testimoni antichi; in effetti già a una prima analisi sommaria se ne notano di apparte-

\footnotetext{
${ }^{82}$ A. Pietersma, in Journal of Biblical Literature 104 (1985) pp. 305-311.

${ }^{83}$ Cañas Reíllo, «Libro de Job», p. 428; Cox, Iob, p. 671b; M. Kepper e M. Witte, Job. Das Buch Ijob (Hiob), in Septuaginta Deutsch. Das griechische Alte Testament in deutscher Übersetzung, eds. W. KRAUS e M. KARRER (Stuttgart 2009) pp. 1007-1056: 1010a-b.

${ }^{84}$ Sulle tecniche di traduzione di questa addizione a OG rimando al noto e ancora autorevole contributo di H. Heater, A Septuagint Translation Tecnique in the Book of Job (Washington, DC, 1982) pp. 31-36. Sulla scorta di P. Dhorme, Le livre de Job (2a ed., Paris 1926) p. ClXII, e soprattutto di S. R. Driver e G. B. Gray, The Book of Job (Edinburgh 1921) p. lxxiii; G. B. GRAY, «The Additions in the Ancient Greek Version of Job», The Expositor 19 (1920) pp. 422-438, Heater crede che l'aggiunta degli stichi del v. 9a-e sia attribuibile a una mano più tarda del traduttore originario, e adduce come conferma il vocabolario qui usato rispetto a OG. Nell'aggiunta infatti individua ben cinque parole che non figurano mai in Giobbe se non negli stichi supplementari del v. 9, insieme a ben tre hapax legomena.
} 
nenti a manoscritti e codici non tardivi, e a Padri come il Crisostomo. Il fatto assume un'importanza non trascurabile; infatti queste lezioni, se molto antiche e molto attestate, potrebbero realmente appartenere a $\mathrm{OG}$, cioè essere entrate nel greco durante la traduzione originaria, o comunque poco dopo, forse anche per rivalutare l'immagine e il ruolo della moglie di Giobbe, e così sottolineare la singolarità della scelta di fedeltà del marito.

Anche Clines ${ }^{85}$ crede che il v. 9a-e sia da considerarsi un'espansione testuale, esempio di una tendenza del midrash di aggiungere qualche dettaglio sui personaggi minori e di elaborarne dei brevi discorsi. In particolare egli precisa -sulla scorta di Duhm- che il nostro passo potrebbe essere effettivamente un' aggiunta secondaria a OG, dato che alle parole greche che qui rendiamo con «ti manterrai saldo nella tua integrità / persevererai» (v. 9b) e «Benedici (= Maledici)» (v. 9e), corrispondono nei vv. 3 e 4 altri vocaboli greci, che vengono resi con il medesimo significato. Clines ne evince che la presentazione positiva della moglie di Giobbe in questa rilettura midrashica del v. 9a-e potrebbe riflettere la tendenza tipica del giudaismo di ritrarre questa donna sotto una luce simpatica e perciò benevola.

\section{DUE DIVERSI RITRATTI}

L'esame di entrambe le tradizioni testuali di Gb 2,9-10 ha permesso di delineare due ritratti differenti, se non opposti, della moglie di Giobbe, o perlopiù ha tratteggiato diversamente la sua funzione a fianco del marito nell' ora in cui egli è messo alla prova.

Il TM ha dato un quadro più negativo della donna e, in linea con la tradizione, ne ha sottolineato il ruolo di tentatrice, dato che ella spinge Giobbe fino alla blasfemia, cioè a maledire Yhwh per la sofferenza che gli è stata ingiustamente inflitta. In tal senso, comunque si voglia leggere l'esortazione בָּרָּ del TM, attenuandone o meno la portata, esso costituisce una chiara sollecitazione al male, fatto che pone l'intervento della moglie sotto una luce certamente negativa.

\footnotetext{
${ }^{85}$ CLINES, Job, p. 53.
} 
Se si passa invece a OG e alla sua aggiunta nel v. 9a-e la prospettiva comincia a mutare e ad aprirsi a una considerazione più positiva. Lo attestano anzitutto le parole che la moglie usa nel v. 9e; esse non sono più nemmeno un invito formale alla bestemmia, ma assumono una formulazione più sfumata, che si presta a una lettura più benevola. In effetti la maledizione è sostituita da un'esortazione accorata, che potrebbe essere resa così: «Di' una parola al Signore»! La conferma viene dalla preposizione $\varepsilon i \varsigma$, premessa a xúpıov, il cui senso più proprio è «verso, a» ${ }^{86}$, non quello di «contro», sebbene sia assodata nel greco biblico una certa intercambiabilità tra le preposizioni, causa di qualche confusione del loro significato ${ }^{87}$. Non è possibile, del resto, ipotizzare qui -come nel TM- un eufemismo, dato che la considerazione favorevole del ruolo della donna è ben definita e precisata ulteriormente grazie all'aggiunta del v. 9a-e. Quindi in OG il senso delle parole della moglie potrebbe essere quello di esortare Giobbe a gridare al Signore, nella sua disperazione di innocente, perseguitato ingiustamente, per cercare rifugio in lui, pur sapendo che un tale ardire potrebbe anche comportare la morte come punizione. Senza certo volerne esagerare la portata, è pur vero che questa linea interpretativa è suffragata proprio dall'espansione testuale degli stichi supplementari del v. 9a-e: in essi il ruolo della moglie è presentato in una sorta di agiografia oleografica, che farebbe pensare a un intervento riabilitativo da parte del traduttore, il quale potrebbe avere colto e riproposto così le intenzioni originarie dell'autore.

In definitiva, per comprendere come considerare correttamente il ruolo della moglie di Giobbe, più che chiedersi se la luce negativa proiettata su di essa dipenda dalla misoginia della tradizione sapienziale, o se il testo biblico sopporterebbe letture di stampo politico o sociologico proprie per sé della modernità, si dovrebbe semmai appurare anzitutto da quale delle due tradizioni testuali esaminate è meglio rappresentato l'Ur-text. Il problema critico si rivela certamente di non facile soluzione, tanto che, almeno per ora, sembra preferibile lasciare affiancati i due distinti ritratti

\footnotetext{
${ }^{86}$ J. Lust, E. Eynikel e K. Hauspie (eds.), Greek-English Lexicon of the Septuagint.

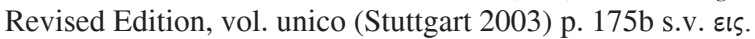

${ }^{87}$ M. Zerwick, Grecitas biblica Novi Testamenti exemplis illustratur (5 $5^{\mathrm{a}}$ ed., Roma 1966) pp. 32-33, parr. 97-98.
} 
della moglie così individuati. Infatti le opposte spiegazioni considerate riflettono due diverse situazioni storiche, con problematiche molto differenti e complesse, che influirono anche sensibilmente sulle riletture attuate durante le fasi della trasmissione del testo e della sua traduzione in greco, la quale -si è visto- fu attività letteraria a tutti gli effetti, dato che l'apporto del traduttore fu sostanzialmente un'interpretazione più che una mera versione dall'ebraico.

Recibido: 11/09/2015

Aceptado: 18/10/2015 